\title{
Unraveling the secrets of Alzheimer's $\beta$-amyloid fibrils
}

\author{
Lynmarie K. Thompson*
}

Department of Chemistry, University of Massachusetts, Amherst, MA 01003

$\mathrm{P}$ roteins adopt an amazing array of sequence-dependent structures that enable them to perform the many chemical functions critical to life. Over the past decade, however, it has become clear that many different protein sequences can also form misfolded, insoluble aggregates known as amyloid fibrils, with common structural elements. Amyloid fibrils appear to be involved in a number of diseases, including Alzheimer's disease, Parkinson's disease, Huntington's disease, prion diseases, and type II diabetes (1). Even ordinary globular proteins (e.g., myoglobin) can form amyloid fibrils under certain conditions (2), suggesting that fibril formation is a previously unappreciated general property of many proteins. Thus, it is of fundamental interest to understand how so many different protein sequences can adopt this alternative structure and it is of medical interest to understand and control disease-related amyloid formation. Knowledge of the three-dimensional structure of amyloid fibrils is critical for understanding the mechanism of fibrillogenesis and for design of possible inhibitors. Unfortunately, amyloid fibrils are noncrystalline and insoluble, and therefore are not amenable to $\mathrm{x}$-ray crystallography and solution NMR, the classic tools of structural biology. In a recent issue of PNAS, Petkova et al. (3) report a structural model for Alzheimer's $\beta$-amyloid fibrils deduced primarily from solid-state NMR experiments. This work provides a significant step forward in understanding $\beta$-amyloid formation and showcases the power of solid-state NMR for obtaining structural information on important but challenging biomolecules.

Amyloid fibrils share a number of characteristics, including a cross- $\beta$ structural motif (1). X-ray fiber diffraction data indicate that the fibrils contain $\beta$-strands that are perpendicular to the fiber axis, with interstrand hydrogen bonding parallel to the fiber axis. However, fiber diffraction cannot determine the chemical details that are needed to understand fibrillogenesis, which parts of the sequence form the $\beta$-strands and which specific amino acid residues are interacting, and cannot even determine whether the proteins in the fibril adopt a unique, ordered structure. This information has now been deduced by Petkova et al. for Alzheimer's $\beta$-amy- loid fibrils using a variety of solid-state NMR approaches (3).

Solid-state NMR methods are well suited to high-resolution structural measurements on noncrystalline solids. Orientation-dependent spin interactions, which are averaged by rapid tumbling in NMR spectra of molecules in solution, lead to broad resonances in NMR spectra of solids. Solid-state NMR experiments typically employ one of two strategies to narrow the resonances and

\section{Amyloid fibrils are noncrystalline and insoluble, and therefore not amenable to $x$-ray crystallography and solution NMR.}

measure structural constraints, $(i)$ preparing oriented samples (e.g., stacks of membrane sheets with uni-axial order), or (ii) using magic angle spinning (MAS) to average orientation-dependent interactions (the approach used by Petkova et al.). There have been significant advances in solid-state NMR studies of biomolecules over the past decade, in both the methodology and its application to important systems. Studies of oriented samples have progressed from the first high-resolution structure of a membrane-bound peptide channel (4), which was determined from multiple samples each isotopically labeled at one residue, to the development of techniques that allow the determination of the backbone structure and orientation of a membrane-spanning $\alpha$-helix by using a single uniformly ${ }^{15} \mathrm{~N}$-labeled sample $(5,6)$. MAS experiments have demonstrated remarkably narrow line widths on microcrystalline samples (7), enabling the resolution and assignment of nearly all of the ${ }^{1} \mathrm{H},{ }^{13} \mathrm{C}$, and ${ }^{15} \mathrm{~N}$ resonances in a single uniformly labeled solid protein sample of a 62-residue $\mathrm{SH} 3$ domain (8). These are promising developments in the pursuit of efficient methodologies for complete structure determination. In addition, studies of specifically labeled samples continue to provide key insights into structure and function: a number of MAS techniques for measuring selected distances and torsion angles have been developed and are being applied to characterize enzyme active sites and to probe structure and mechanism in membrane proteins (see ref. 9 for a review of recent MAS applications). The work of Petkova et al. (3) is an example of this latter approach.

The proposed structural model for Alzheimer's $\beta$-amyloid fibrils is based on a large number of strategically chosen constraints, demonstrating an excellent approach for using solid-state NMR to determine structures of other peptidebased systems. The strategy used by Petkova et al. (3) was to characterize the full-length 40-residue polypeptide by using a combination of many low-precision constraints (from linewidths and chemical shifts) and a smaller number of high-precision constraints (from measured distances and torsion angles). The authors have designed a highly efficient means of collecting this extensive set of constraints. A fully uniformly labeled sample cannot be used because linenarrowing by MAS yields 1.5- to 2.5ppm line widths for noncrystalline ordered solids, which means chemically equivalent spins (e.g., multiple Gly residues) typically have unresolved resonances. Therefore, Petkova et al. synthesized four peptide samples, which each incorporated five to seven residues with uniform ${ }^{13} \mathrm{C}$ and ${ }^{15} \mathrm{~N}$ labeling. The labeled sites were chosen so that two-dimensional correlation spectra of each sample could be unambiguously assigned (i.e., five to seven different residues with distinct chemical shift patterns were chosen). This strategy yielded linewidths and chemical shifts for nearly all of the carbons and nitrogens from 22 residues throughout the protein. Linewidths were used to determine which segments adopt a unique structure: residues $1-10$ are disordered and residues 12-36 are ordered. Chemical shifts were analyzed based on comparisons with chemical shifts in proteins of known structures to determine the backbone secondary structure: residues $12-24$ and 30-40 adopt $\beta$-strand backbone conformations. These data identified a region of non- $\beta$ structure (residues 25-29) between the

See companion article on page 16742 in issue 26 of volume 99 .

*E-mail: thompson@chem.umass.edu. 


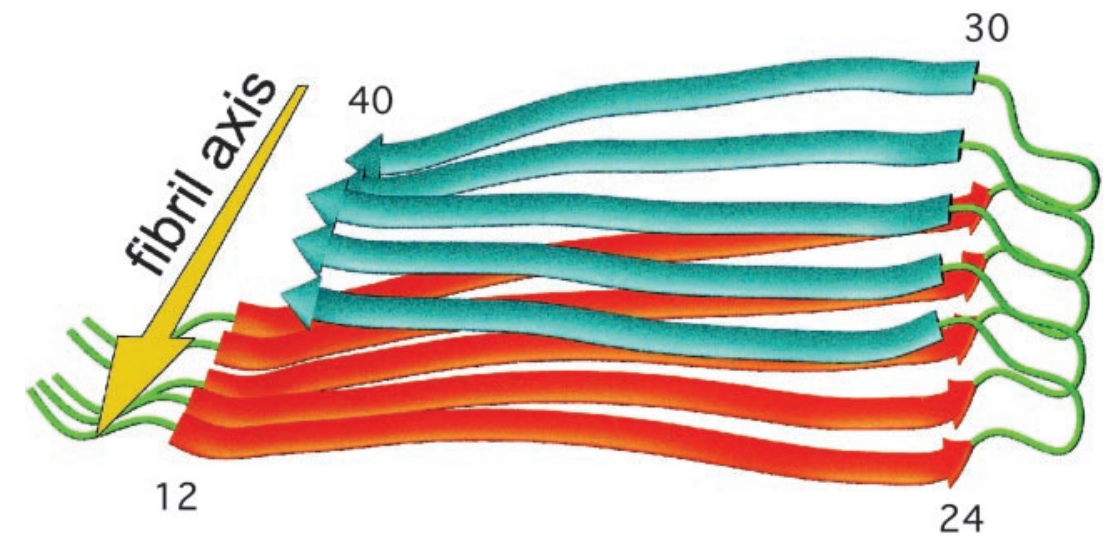

Fig. 1. Schematic representation of the proposed cross- $\beta$ unit of amyloid fibrils formed by the full-length Alzheimer's peptide $A \beta_{1-40}$. The first eight residues (not shown) are disordered; the two $\beta$-strands (residues 12-24 and 30-40) are represented by arrows and flank a bend that is stabilized by a salt bridge between residues 23 and 28. Six peptides are shown, which interact via backbone hydrogen bonds between the $\beta$-strands to form a double layer of parallel $\beta$-sheets ( $\mathrm{H}$ bonds are parallel to the fibril axis). The bottom face of the red $\beta$-sheet is polar; the top face of the blue $\beta$-sheet is hydrophobic and proposed to interact with the corresponding face of another cross- $\beta$ unit to form the narrowest observed fibrils. Adapted from Petkova et al. (3) with permission.

two $\beta$-strands that was further characterized by using more advanced NMR methods to determine the backbone torsion angles. In addition, the mode of intermolecular interactions between the peptides has previously been established by this group using solid-state NMR measurements of interstrand distances: each of the two $\beta$-strands forms an in-register parallel $\beta$-sheet with the corresponding residues in neighboring peptides of the fibril (10). All of these solid-state NMR derived constraints were combined with the cross- $\beta$ motif established by $\mathrm{x}$-ray fiber diffraction to deduce a structural model that has chemically reasonable hydrophobic and electrostatic interactions and is consistent with fibril dimensions observed by electron microscopy (see Fig. 1).

The proposed structure of Petkova et al. (3) is a notable advance in our understanding of the structure of Alzheimer's $\beta$-amyloid fibrils. The insoluble nature of these fibrils has made it difficult to obtain detailed structural information and constraints for developing structural models. The first solid-state NMR study of Alzheimer's $\beta$-amyloid fibrils (11) and a more recent study (12) focused on the small peptide fragments $A \beta_{34-42}$ (residues 3442 ) and $\mathrm{A} \beta_{16-22}$, respectively, and concluded that these fragments adopt an antiparallel $\beta$ structure in the fibrils. Solidstate NMR studies of longer peptides $\mathrm{A} \beta_{10-35}$ and $\mathrm{A} \beta_{1-40}$ have demonstrated that these peptides adopt parallel, in-register $\beta$ structures $(10,13)$. This structural variation with peptide length means that a structural model for the amyloid fibrils observed in vivo, in which the peptide length ranges from 39 to 43 amino acids, must be deduced from constraints mea- sured on full-length peptides and should incorporate parallel $\beta$-strand interactions. Solid-state NMR studies of $\mathrm{A} \beta_{10-35}$ were first to demonstrate the parallel, in-register $\beta$ structure (13). Tycko and coworkers confirmed that this structure is retained in fibrils formed by the full-length peptide $\mathrm{A} \beta_{1-40}(10)$ and have now established the presence of a bend between two $\beta$-strand regions (3). In a recent molecular dynamics study, Ma and Nussinov (14) compared possible structures of fibrils formed by different length peptides and independently proposed a bend in the structure of fibrils formed by a long peptide $\left(\mathrm{A} \beta_{10-35}\right)$, resulting in a double layered sheet very similar to the structure proposed by Petkova et al.

Although amyloid fibrils share in common the cross- $\beta$ motif, the structural details are likely to vary for each sequence. As described above, studies of Alzheimer's amyloid fibrils have demonstrated that subsets of the same sequence form fibrils with significantly different structures (antiparallel vs. parallel $\beta$ strand interactions). Structural studies of amyloid fibrils formed by other proteins are needed so that comparisons between structural models can reveal general principles regarding which specific interactions stabilize the structures and may be important in promoting fibril formation. Randomly generated polypeptide sequences with alternating hydrophobic and hydrophilic residues have been shown to form fibrils (15). Such sequences are under-represented in natural protein sequences, suggesting that perhaps evolution has selected against sequences prone to amyloid fibril formation (15). Investigations of fibrillogenesis have shown that fibril formation is favored by conditions that populate partially folded intermediates of proteins (1). Further understanding of both fibril structures and the mechanism of formation will test ideas of how protein sequences have evolved to minimize amyloid fibril formation. Such knowledge is critical for understanding protein folding, and for informing efforts to engineer or design useful proteins.

The impact of this type of work on the prevention or treatment of amyloid diseases depends on the results of ongoing studies to clarify the role of amyloid fibrils in these diseases. It is still not clear whether amyloid fibrils are the causative agent of the neurodegeneration in Alzheimer's and Parkinson's disease $(16,17)$. One problem with this hypothesis is that the quantity of amyloid fibrils found in the brain apparently does not correlate with the severity of the symptoms. In addition, recent studies of an $\alpha$-synuclein mutant protein linked to early onset Parkinson's disease have shown that the protein has increased rates of oligomerization and decreased rates of fibrillization (18). This protein and other mutants linked to Parkinson's and Alzheimer's disease have been shown to form a pore-like species that could form cytotoxic channels in membranes (17). Clearly it is critical to determine whether it is the fibril or a precursor of the fibril that is the toxic agent in each disease, to know whether treatment should aim to inhibit or accelerate fibril formation. This information combined with knowledge of the mechanism of formation and of the structures of each species could be used to design molecules to inhibit or promote formation of fibrils.

Structural biologists have deposited the coordinates for the structures of over 19,000 biomolecules in the Protein Data Bank (PDB). Such structures have long been essential for understanding the fundamental mechanisms of biomolecules and have more recently enabled the success of many rational drug design efforts. However, many medically important biomolecules remain structurally uncharacterized because they are resistant to x-ray crystallography and solution NMR. The contents of the PDB are biased toward the systems most amenable to these techniques, proteins that are stable and easily crystallized or small. Therefore, entire classes of proteins are under-represented in the PDB, including membrane proteins, dynamic proteins, and possibly new classes not yet identified. The work of Petkova et al. (3) demonstrates the promise of solid-state NMR for structural characterization of noncrystalline solids. This approach makes it possible to obtain highresolution structural information on medically important but challenging biomolecules, such as amyloid fibrils and membrane proteins. 
1. Rochet, J. C. \& Lansbury, P. T., Jr. (2000) Curr. Opin. Struct. Biol. 10, 60-68.

2. Fandrich, M., Fletcher, M. A. \& Dobson, C. M. (2001) Nature 410, 165-166.

3. Petkova, A. T., Ishii, Y., Balbach, J. J., Antzutkin, O. N., Leapman, R. D., Delaglio, F. \& Tycko, R. (2002) Proc. Natl. Acad. Sci. USA 99, 16742-16747.

4. Ketchem, R. R., Hu, W. \& Cross, T. A. (1993) Science 261, 1457-1460.

5. Marassi, F. M. \& Opella, S. J. (2000) J. Magn. Reson. 144, 150-155.

6. Wang, J., Denny, J., Tian, C., Kim, S., Mo, Y., Kovacs, F., Song, Z., Nishimura, K., Gan, Z., Fu, R., et al. (2000) J. Magn. Reson. 144, 162-167.

7. McDermott, A., Polenova, T., Bockmann, A., Zilm, K. W., Paulsen, E. K., Martin, R. W. \&
Montelione, G. T. (2000) J. Biomol. NMR 16, 209-219.

8. Pauli, J., Baldus, M., van Rossum, B., de Groot, H. \& Oschkinat, H. (2001) Chembiochem 2, 272-281.

9. Thompson, L. K. (2002) Curr. Opin. Struct. Biol. 12, 661-669.

10. Balbach, J. J., Petkova, A. T., Oyler, N. A., Antzutkin, O. N., Gordon, D. J., Meredith, S. C. \& Tycko, R. (2002) Biophys. J. 83, 1205-1216.

11. Lansbury, P. T., Costa, P. R., Griffiths, J. M., Simon, E. J., Auger, M., Halverson, K. J., Kocisko, D. A., Hendsch, Z. S., Ashburn, T. T., Spencer, R. G. S., et al. (1995) Nat. Struct. Biol. 2, 990-998.

12. Balbach, J. J., Ishii, Y., Antzutkin, O. N., Leapman, R. D., Rizzo, N. W., Dyda, F., Reed, J. \& Tycko, R. (2000) Biochemistry 39, 13748-13759.
13. Benzinger, T. L., Gregory, D. M., Burkoth, T. S., Miller-Auer, H., Lynn, D. G., Botto, R. E. \& Meredith, S. C. (1998) Proc. Natl. Acad. Sci. USA 95, 13407-13412.

14. Ma, B. \& Nussinov, R. (2002) Proc. Natl. Acad. Sci. USA 99, 14126-14131.

15. West, M. W., Wang, W., Patterson, J., Mancias, J. D., Beasley, J. R. \& Hecht, M. H. (1999) Proc. Natl. Acad. Sci. USA 96, 11211-11216.

16. Hardy, J. \& Selkoe, D. J. (2002) Science 297, 353-356.

17. Lashuel, H. A., Hartley, D., Petre, B. M., Walz, T. \& Lansbury, P. T., Jr. (2002) Nature 418, 291.

18. Conway, K. A., Lee, S. J., Rochet, J. C., Ding, T. T., Williamson, R. E. \& Lansbury, P. T., Jr. (2000) Proc. Natl. Acad. Sci. USA 97, 571-576. 